\title{
Neuritic Deposition of Agrin on Culture Substrate: Implications for Nerve-Muscle Synaptogenesis
}

\author{
M. W. Cohen, ${ }^{1}$ F. Moody-Corbett, ${ }^{2}$ and E. W. Godfrey ${ }^{3}$ \\ 'Department of Physiology, McGill University, Montreal, Quebec, Canada H3G 1Y6, '2Division of Basic Medical Sciences, \\ Memorial University, St. John's, Newfoundland, Canada A1B 3V6, and 3Department of Cellular Biology and Anatomy, \\ Medical College of Wisconsin, Milwaukee, Wisconsin 53226
}

Recent experiments have indicated that neural agrin is deposited at newly forming nerve-muscle synapses and has a primary synaptogenic role there. As a step toward assessing how the spatial arrangement of new synaptic sites is regulated, we compared the pattern of agrin deposition by Xenopus neurites on culture substrate and on muscle cells. The neurons were grown on a substrate that bound their externalized agrin so tightly that it remained bound even when the neurites retracted spontaneously or were eliminated experimentally. By contrast, the neural cell adhesion molecule, NCAM, was not left behind on the substrate when the neurites were eliminated. Agrin, visualized by immunofluorescent staining, was deposited on the culture substrate in a continuous fashion along virtually the entire neuritic arbor of many spinal cord (SC) neurites. The pattern of agrin deposition by the same neurites changed from continuous to discontinuous when the neurites contacted muscle cells, and it became continuous again when the neurites returned to the culture substrate. The sites of agrin deposition on muscle cells were also sites of accumulation of $\mathrm{ACh}$ receptors (AChRs). Dorsal root ganglion (DRG) neurons and some SC neurons did not deposit agrin along their neuritic outgrowth, either on the culture substrate or on the muscle cells, and did not induce AChR accumulation at sites of contact with muscle cells. Besides adding to the evidence in support of agrin's synaptogenic role, the findings indicate that muscle cells significantly influence how neural agrin and synaptic sites become distributed along paths of neurite-muscle contact.

IKey words: agrin deposition, culture substrate, Xenopus, spinal cord neurons, dorsal root ganglion neurons, nervemuscle synaptogenesis, ACh receptors, neural cell adhesion molecule]

The factors and mechanisms that participate in regulating the distribution of synaptic sites on target cells are of central importance to an understanding of synaptogenesis, but remain largely unresolved at present. For the case of embryonic muscle cells, it is known from studies in culture that they can develop

\footnotetext{
Received June 29, 1993; revised Nov. 4, 1993; accepted Nov. 24, 1993.

This work was supported by MRC (M.W.C., F.M.-C.) and NIH (NS27218, E.W.G.). We thank G. Hébert and D. McDonald for expert technical assistance Correspondence should be addressed to M. W. Cohen, Department of Physiology, McGill University, 3655 Drummond Street, Montreal, Quebec, Canada H3G 1 Y 6 .

Copyright (C) 1994 Society for Neuroscience $0270-6474 / 94 / 143293-1$ I $\$ 05.00 / 0$
}

postsynaptic-like specializations even in the absence of innervation or previous innervation in vivo. These specializations include an aggregation of two functionally important proteins in synaptic transmission, $\mathrm{ACh}$ receptors (AChRs) and $\mathrm{AChE}$ (Moody-Corbett and Cohen, 1981). Yet the neurites of embryonic neurons do not seek out these sites, nor do they appear to follow any pathway that might have been preestablished on the muscle cells. Rather, the neurites take unpredictable routes as they grow across muscle cells and they induce new synaptic specializations along their path of contact with muscle while at the same time inhibiting the formation and survival of postsynaptic-like specializations elsewhere on the muscle cells (Anderson and Cohen, 1977; Frank and Fischbach, 1979; MoodyCorbett and Cohen, 1982). Of particular interest is that the resulting synaptic sites occupy on average only about $25 \%$ or less of the pathway of neuritic growth on individual muscle cells (Role et al., 1985; Cohen et al., 1987). Typically these pathways exhibit a pattern of discrete synaptic sites, usually less than 20 $\mu \mathrm{m}$ in length and separated from each other by gaps that vary from as little as $2 \mu \mathrm{m}$ to more than $20 \mu \mathrm{m}$ (see Fig. 8). Such discontinuities occur even under conditions where competitive interactions between neurons (Cohen et al., 1987) and synaptic transmission (Anderson and Cohen, 1977; Anderson et al., 1977) have been eliminated.

In the present study we have attempted to evaluate the relative influence of neurites and of muscle cells in generating this discontinuous pattern by comparing the pattern of neuritic deposition of agrin on and off muscle cells. Neural agrin, a $200 \mathrm{kDa}$ protein, appears to be a primary synaptogenic agent in the nerveinduced aggregation of AChRs at embryonic nerve-muscle synapses (McMahan et al., 1992). Part of the evidence is that agrin is supplied by spinal cord (SC) neurites to the synapses they form on embryonic muscle cells and is present even at the earliest-detectable nerve-induced microaggregates of AChRs (Cohen and Godfrey, 1992). In addition appropriate anti-agrin antibodies inhibit nerve-induced clustering of AChRs (Reist et al., 1992). Furthermore, as described in the present study, neurons that are incompetent to induce AChR aggregation do not externalize detectable amounts of agrin along their neuritic arbor.

In view of the evidence in support of agrin's synaptogenic role, the way in which agrin is externalized by neurites and how it is handled by muscle cells are likely to be important factors in determining the spatial distribution of synaptic sites. For example, the interrupted pattern of synaptic sites on individual muscle cells might arise if the pattern of neuritic externalization of agrin were discontinuous. To address the latter possibility, 
we grew neurons on a culture substrate that binds agrin tightly, so that the distribution of the agrin they externalized along their neuritic outgrowth could be visualized directly. Our findings reveal that competent $\mathrm{SC}$ neurites deposit agrin on the culture substrate in a continuous fashion, in contrast to the discontinuous pattern seen on muscle cells. It follows that muscle cells exert a significant influence on how neural agrin and its associated synaptic sites become distributed along paths of neuritemuscle contact.

Some of the findings have been reported briefly in an abstract (Cohen et al., 1992).

\section{Materials and Methods}

Culture suhstrate. The substrate consisted of a combination of rat tail collagen and mouse tumor entactin, collagen, and laminin (ECL; Upstate Biotechnology, Inc., Lake Placid, NY) on glass coverslips. The rat tail collagen was applied as a thin film and allowed to dry. After assembling the culture chamber (see Anderson et al., 1977) the ECL solution was applied at a protein concentration of $5-20 \mu \mathrm{g} / \mathrm{ml}$ for $30-60 \mathrm{~min}$. Culture chambers were then rinsed with $67 \%(\mathrm{v} / \mathrm{v})$ L15 and refrigerated until used, usually $1 \mathrm{~d}$ later.

Cultures. Spinal cords were isolated from 1-d-old Xenopus laevis embryos (stages 22-26; Nieuwkoop and Faber, 1967) and then dissociated and plated as described previously (Cohen et al., 1987). When required, myotomal muscle cells were also obtained from these embryos. For some experiments similar procedures were used to isolate, dissociate, and plate presumptive SC neurons from the caudal portion of the neuroectoderm of stage 13-14 embryos. DRG cultures were also prepared according to the procedures described previously (Cohen and Weldon, 1980).

In initial experiments the culture medium consisted of $67 \% \mathrm{~L} 15$ and $0.2 \mu \mathrm{g} / \mathrm{ml}$ Holmes $\alpha-1$ protein, as in previous studies (sec Cohen et al., 1987). Subsequently, when the latter protein was no longer available commercially, the culture medium consisted of $67 \% \mathrm{~L} 15,0.25 \%(\mathrm{v} / \mathrm{v})$ dialyzed horse serum, and $1 \%(\mathrm{v} / \mathrm{v})$ Ultraser-G. In a few experiments $67 \% \mathrm{~L} 15$ was used by itself. The new media did not appear to alter the neuritic deposition of agrin. All components of the culture media were obtained from GIBCO-Bethesda Research Laboratories (Burlington, Ontario, Canada)

Most experiments were carried out on 1-d-old cultures, but similar results were obtained with 2 - and 3-d-old cultures.

Elimination of neurites. To eliminate the neurons and their neurites, cultures were first rinsed aggressively by applying a stream of $67 \% \mathrm{~L} 15$ through a 20 gauge needle positioned about $1 \mathrm{~cm}$ above the cells. The turbulence generated by this rinsing procedure eliminated all-neurons and virtually all their neurites, although some neuritic remnants sometimes remained adherent to the substrate. Some flat non-neuronal cells, including pigmented cells, also survived this procedure. The cultures were then treated with $1 \%(\mathrm{v} / \mathrm{v})$ Triton X-100 for $10 \mathrm{~min}$ and rinsed well with $67 \% \mathrm{~L} 15$. This eliminated all neuritic remnants as well as the non-neuronal cells. Externalized, substrate-bound agrin survived this neurite elimination procedure (see Results), even when the concentration of Triton X-100 was increased to $5 \%$. Treatment with Triton X-100 without prior aggressive rinsing was also effective in eliminating all neurons and their neurites but left many intracellular organelles adherent to the substrate. Accordingly, it was preferable to precede the Triton $\mathrm{X}-100$ treatment with aggressive rinsing.

Antibodies. Rabbit antiserum 36 (anti-36; Godfrey, 1991) and mouse ascites monoclonal antibodies C3 and F11 (Godfrey et al., 1988) were used to stain Xenopus agrin (Cohen and Godfrey, 1992). Preimmune serum (pre-36) from the same rabbit served as a control, as did mouse ascites monoclonal antibody $\mathrm{B} 7$, which is known to be reactive with chick agrin (Godfrey et al., 1988) but proved to be unreactive with Xenopus agrin. The sera and ascites fluid were used at dilutions of 1:200 to $1: 400$.

Primary antibodies directed against intracellular proteins in neurons were also used. One was a rabbit antiserum against tau $(\tau)$, a microtubule-associated protein. Anti- $\tau$ (Sigma Chemical Co., St. Louis, MO) was effective at a dilution of $1: 1000$. Mouse ascites monoclonal antibody 48 directed against synaptotagmin (also known as p65; Matthew et al., 1981; Südhof and Jahn, 1991), was used at dilutions of 1:100 to 1:250 as described previously (Cohen et al., 1987). The antibody was kindly provided by L. F. Reichardt (Neuroscience Unit, University of California at San Francisco). Mouse ascites monoclonal antibody SMI-31 (Sternberger Monoclonals Inc., Jarrettsville, MD) against neurofilament protein was effective at dilutions of 1:5000.

For immunofluorescent staining of NCAM, we used the IgG fraction $(2-10 \mu \mathrm{g} / \mathrm{ml})$ of a rabbit antiserum known to be reactive with Xenopus NCAM, particularly the $180 \mathrm{kDa}$ and $140 \mathrm{kDa}$ transmembrane isoforms (Jacobson and Rutishauser, 1986). The antibody was kindly provided by U. S. Rutishauser (Case Western Reserve University, Cleveland, $\mathrm{OH})$.

Affinity-purified fluorescein (F)- and rhodamine (R)-conjugated goat anti-rabbit (GAR) and goat anti-mouse (GAM) immunoglobulins were used as secondary antibodies to visualize the binding sites of the primary antibodies. FGAR and FGAM were obtained from Organon TeknikaCappel and from Molecular Probes. RGAR and RGAM were obtained from Molecular Probes. All secondary antibodies were used at 10-20 $\mu \mathrm{g} / \mathrm{ml}$.

Primary and secondary antibodies were diluted in a solution of $67 \%$ L15 and 1\% goat serum, and were filtered (Millex GV, Millipore; 0.22 $\mu \mathrm{m}$ pore size) before use.

Immunofluorescent staining. Living cultures were stained for agrin at $\sim 6^{\circ} \mathrm{C}$, as described previously (Cohen and Godfrey, 1992), or at room temperature $\left(23-25^{\circ} \mathrm{C}\right)$. These differences in temperature did not appear to affect the pattern or intensity of the agrin immunofluorescence. Brief$\mathrm{ly}$, the staining protocol at room temperature included preincubation in a solution of $67 \% \mathrm{~L} 15$ and $1 \%$ goat serum for 5-10 min followed by incubation in the primary antibody for $30 \mathrm{~min}$ and in the secondary antibody for $30 \mathrm{~min}$. After each antibody the culture was rinsed five times. The cultures were then fixed with $4 \%(w / v)$ formaldehyde as described previously (Cohen et al., 1987) and refrigerated. Subsequently, they were rinsed, cleared with a glycerol solution (Cohen et al., 1987), and stored at $-16^{\circ} \mathrm{C}$ until examined by microscopy.

To stain effectively for intracellular proteins it was necessary first to fix and permeabilize the cultures. This was done by treating the cultures with methanol for $15 \mathrm{~min}$ at $-16^{\circ} \mathrm{C}$ or by fixing with $4 \%$ formaldehyde or $4 \%$ paraformaldehyde for $5 \mathrm{~min}$ followed by rinsing and incubation in $1 \mathrm{mg} / \mathrm{ml}$ saponin for $15 \mathrm{~min}$. When the antibodies against intracellular proteins were tested on living cultures or after neurite elimination their concentration was increased by as much as fivefold.

To visualize the distribution of NCAM on neurites, the cultures were prefixed for $5-10 \mathrm{~min}$ with $4 \%$ formaldehyde or $4 \%$ paraformaldehyde. Prefixation was employed in order to avoid antibody-induced redistribution of NCAM, because it is known that the transmembrane isoforms of NCAM are mobile within the plane of the surface membrane (Pollerberg et al., 1986). After rinsing the fixed cultures, the staining protocol was similar to that described for agrin. In experiments in which the neurites were eliminated subsequent prefixation was unnecessary, and the concentration of anti-NCAM was increased by as much as fivefold.

Cultures were examined with phase-contrast and fluorescence optics as in previous studies (Cohen et al., 1987; Cohen and Godfrey, 1992).

\section{Results}

\section{$S C$ neurites on culture substrate}

As shown in Figure 1, agrin immunofluorescence was observed along much of the neuritic outgrowth of SC neurons. Its intensity varied along different neurites, and was generally in the same range as the agrin immunofluorescence at nerve-muscle synapses in culture (see Fig. 8). Similar patterns were seen whether the cultures were stained alive or after fixation with $4 \%$ formaldehyde or $4 \%$ paraformaldehyde, although the intensity of the immunofluorescence declined with increasing fixation time. Tests described below confirmed that the staining was specific for externalized agrin.

It is apparent from Figure 1 that some portions of the neuritic outgrowth did not exhibit any agrin immunofluorescence (arrowheads in $A$ ) and that there were pathways of agrin immunofluorescence with no corresponding neurites (arrows in $B$ ). The latter observation suggests that neurites deposited agrin at those sites earlier and then retracted, leaving behind the agrin bound to the culture substrate. Such a case of neuritic retraction is evident in Figure 2. The culture was photographed alive (Fig. 
$2 A$ ), stained for agrin, and then fixed and rephotographed (Fig. $2 B)$. Two obvious changes in the neuritic pattern took place during the $2 \mathrm{hr}$ interval between photographing Figure $2 A$ and fixation. Distally the neurite grew about $40 \mu \mathrm{m}$ (arrowheads in $B$ ) and proximally a portion of the original neuritic loop retracted (large arrowhead in $A$ ). Figure $2 C$ shows that agrin immunofluorescence was associated with the original position of the neuritic loop but was absent from the new position of the retracted neurite. This is seen more clearly at higher magnification in Figure 2, $D$ and $E$. A second example of a branch of agrin immunofluorescence without a surviving neurite is indicated by the arrow in Figure $2 C$ and is shown at higher magnification in Figure 2, $F$ and $G$. The higher-magnification observations also reveal that all of the immunofluorescence along the neuritic pathway was in the same plane of focus as the nonspecific dots of fluorescence on the surface of the culture substrate (Fig. 2E, $G, I$ ). The simplest explanation for these observations is that the externalized agrin at the neurite-substrate interface was bound more tightly to the substrate than to the neurites, so that upon neuritic retraction the agrin was left behind.

Agrin immunofluorescence was also observed in the region of new neuritic growth (Fig. $2 B, C, H, I$ ). Typically, as in this example, the immunofluorescence in newly grown regions was less intense than in neighboring proximal regions. It may be that externalized agrin continues to accumulate at a particular site on the substrate for some time after the growth cone has passed that site.

For most experiments neurons were isolated from stage 2226 embryos (approximate age, 24-30 hr; see Nieuwkoop and Faber, 1967) when motor axons have just begun to invade the developing myotomal muscle (Kullberg et al., 1977; Chow and Cohen, 1983). However, previous nerve-muscle contact in vivo proved to be unnecessary for the externalization of agrin by SC neurons in culture. As shown in Figure 3, equally bright and extensive agrin immunofluorescence was seen along the neuritic outgrowth of neurons obtained from stage 13 "neural plate" embryos (approximate age, $15 \mathrm{hr}$ ), some $6 \mathrm{hr}$ prior to any axonal outgrowth in vivo (see Hayes and Roberts, 1973).

In the example of Figure 3, agrin immunofluorescence was associated with virtually the entire neuritic arbor of a single isolated neuron. This was the case for the majority of isolated $\mathrm{SC}$ neurons, but within this group there were differences in the prevailing intensity of the immunofluorescence along the neuritic arbors. Often, as in the example of Figure 3, immunofluorescence was present around the cell body and was relatively faint for some $20-40 \mu \mathrm{m}$ along the most proximal portion of the primary neurites. Additional features, not apparent in Figure 3 , were also noted. Sometimes, just distal to a neuritic branch point there was a 5-10 $\mu \mathrm{m}$ gap in the immunofluorescence along one of the branches. Occasionally, one of the distal neuritic branches, comprising less than $10 \%$ of the entire neuritic arbor, had no immunofluorescence associated with it. Otherwise, the isolated SC neurons that exhibited immunofluorescence did so along their entire neuritic arbor. A second class of isolated SC neurons, which formed the minority, exhibited no immunofluorescence at all along their neuritic arbor. That most of the SC neurons in these cultures externalize agrin is also suggested by the high incidence of agrin immunofluorescence in fields with intermingling neurites from multiple neurons (Fig. 1; see also Fig. 6). These observations correlate well with those of previous studies indicating that most, but not all, SC neurons in these
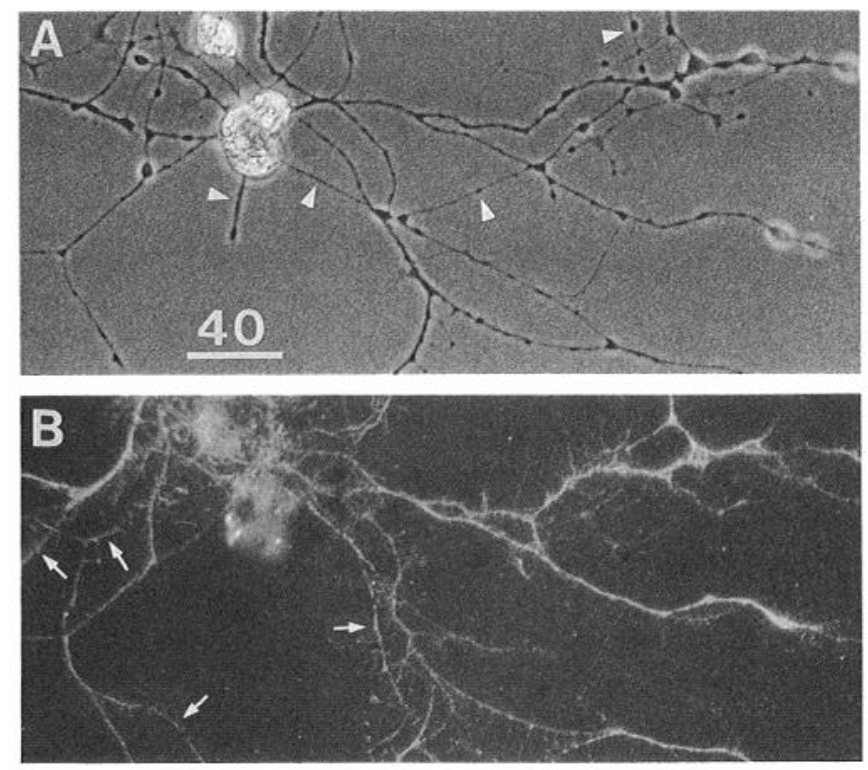

Figure 1. Agrin immunofluorescence along SC neurites. Phase contrast $(A)$ and fluorescence $(B)$ views of the same field show immunofluorescence along much of the neuritic outgrowth. Arrowheads in $A$ point to neurites that did not exhibit detectable immunofluorescence. Arrows in $B$ point to agrin pathways where there were no surviving neurites. Agrin was stained with C3 followed by FGAM. Scale bar, $40 \mu \mathrm{m}$.

cultures are competent to induce clustering of AChRs at sites of neurite-muscle contact and that this capacity extends throughout their neuritic outgrowth (Anderson et al., 1977; Cohen et al., 1987).

The examples of Figures 1-3 illustrate that the agrin immunofluorescence was essentially continuous along neuritic pathways, even when the neurites were a few hundred micrometers long. The width of the immunofluorescence generally varied with the width of the neurite and there was sometimes outlining at the edges, especially at varicosities and other regions where the neurites were broader (Fig. 2C,E; see also Fig. 6). Usually the borders of the immunofluorescence were sharply defined but sometimes they were indistinct and tended to fade over 1-2 $\mu \mathrm{m}$ into the background, suggesting some additional diffusion of the agrin. Occasionally, the immunofluorescence had a mottled appearance (e.g., Fig. $4 F$ ). The basis of these different details in the appearance of the agrin immunofluorescence was not investigated.

\section{Specificity of immunofluorescence}

The immunofluorescence described above was obtained with each of three anti-agrin antibodies (rabbit anti-36 and mouse $\mathrm{C} 3$ and F11) that are known to be reactive with Xenopus agrin (see Cohen and Godfrey, 1992). By contrast, rabbit pre-36 and mouse B7, which is reactive with chick agrin (Godfrey et al., 1988) but not with Xenopus agrin, were ineffective in staining the same neuritic pathways (Fig. 4). In addition, when cultures were stained with a combination of anti-36 and C3 or F11 (followed by a combination of anti-rabbit and anti-mouse second antibodies having contrasting fluorophores) the pattern of immunofluorescence obtained with the polyclonal antibody was identical to that obtained with either of the monoclonal antibodies (see Fig. $6 D, E$ ). It follows that the immunofluorescence obtained with anti-36, C3, and F11 was specific for agrin.

Besides the specific agrin immunofluorescence seen along neu- 

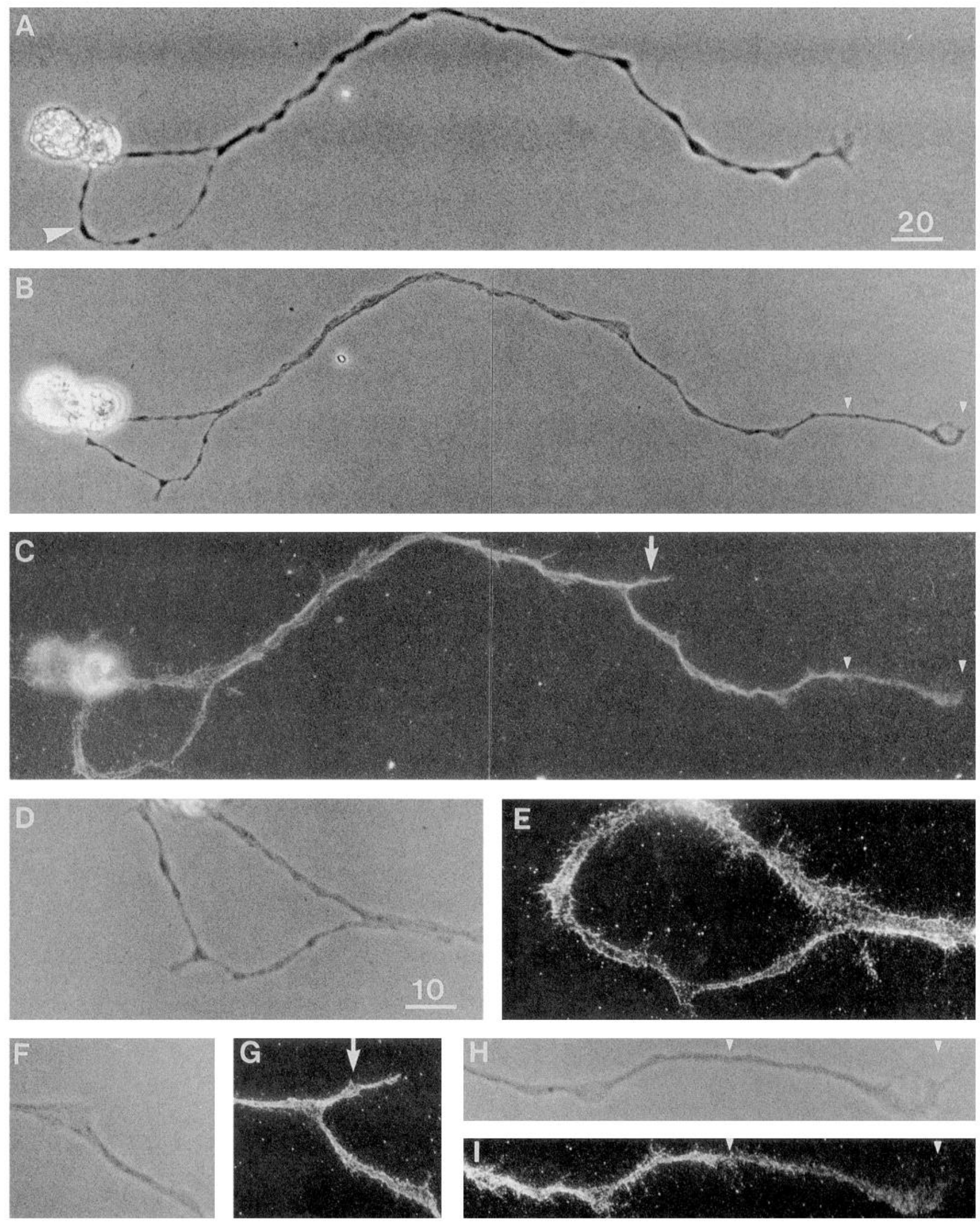

Figure 2. Agrin immunofluorescence following neurite retraction and neurite growth. A, A field in a living culture. Large arrowhead points to a portion of the neuritic pathway that subsequently retracted. $B$, The same field, $2 \mathrm{hr}$ later, after fixation. Prior to fixation the culture was stained with $\mathrm{C} 3$ followed by FGAM. Note the neuritic retraction as well as the region of new growth (small arrowheads). $C$, Immunofluorescence is seen 

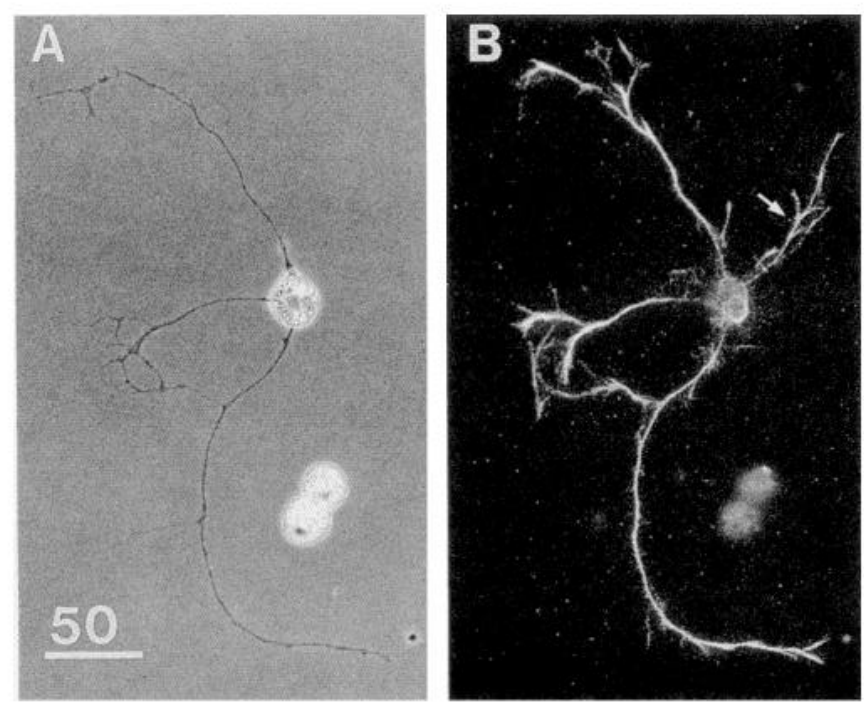

Figure 3. Agrin immunofluorescence along the neuritic outgrowth of a single isolated neuron in a culture prepared from the neuroectoderm of stage 13 embryos. Almost all of the neuritic arbor $(A)$ exhibited immunofluorescence $(B)$. Some of the immunofluorescence not associated with surviving neurites is indicated by the arrow in $B$. Agrin was stained with anti-36 and C3 followed by FGAR and FGAM. Scale bar, $50 \mu \mathrm{m}$.

ritic pathways in the same focal plane as the culture substrate, some bright immunofluorescence was also seen at different focal planes in regions of neuronal cell bodies (Figs. 1, 4). This latter immunofluorescence proved to be nonspecific (Fig. 4) and presumably was associated with adherent cell debris or damaged cells.

\section{External location of immunofluorescence}

Since the cultures were routinely stained prior to fixation it follows that the agrin immunofluorescence was outside rather than inside the neurites. The failure of antibodies to enter living neurites was confirmed in tests with antibodies directed against intracellular proteins including a microtubule-associated protein, a synaptic vesicle protein, and a neurofilament protèin (see Materials and Methods). Each of these three antibodies stained all neurites (agrin positive and agrin negative) brightly when the staining was carried out after fixation and permeabilization, but they were entirely ineffective in staining living neurites even when their concentration was increased fivefold (Fig. 5). In addition, the agrin immunofluorescence along neuritic pathways was observed when the staining was carried out at low temperature (about $6^{\circ} \mathrm{C}$ ) or after fixation (see Fig. 7), conditions that inhibit internalization. These tests, as well as those on neurite elimination described below, support the conclusion that the agrin immunofluorescence was due to externalized agrin.

\section{Substrate binding of externalized agrin}

To test further whether the externalized agrin was bound to the culture substrate, the agrin immunofluorescence was examined
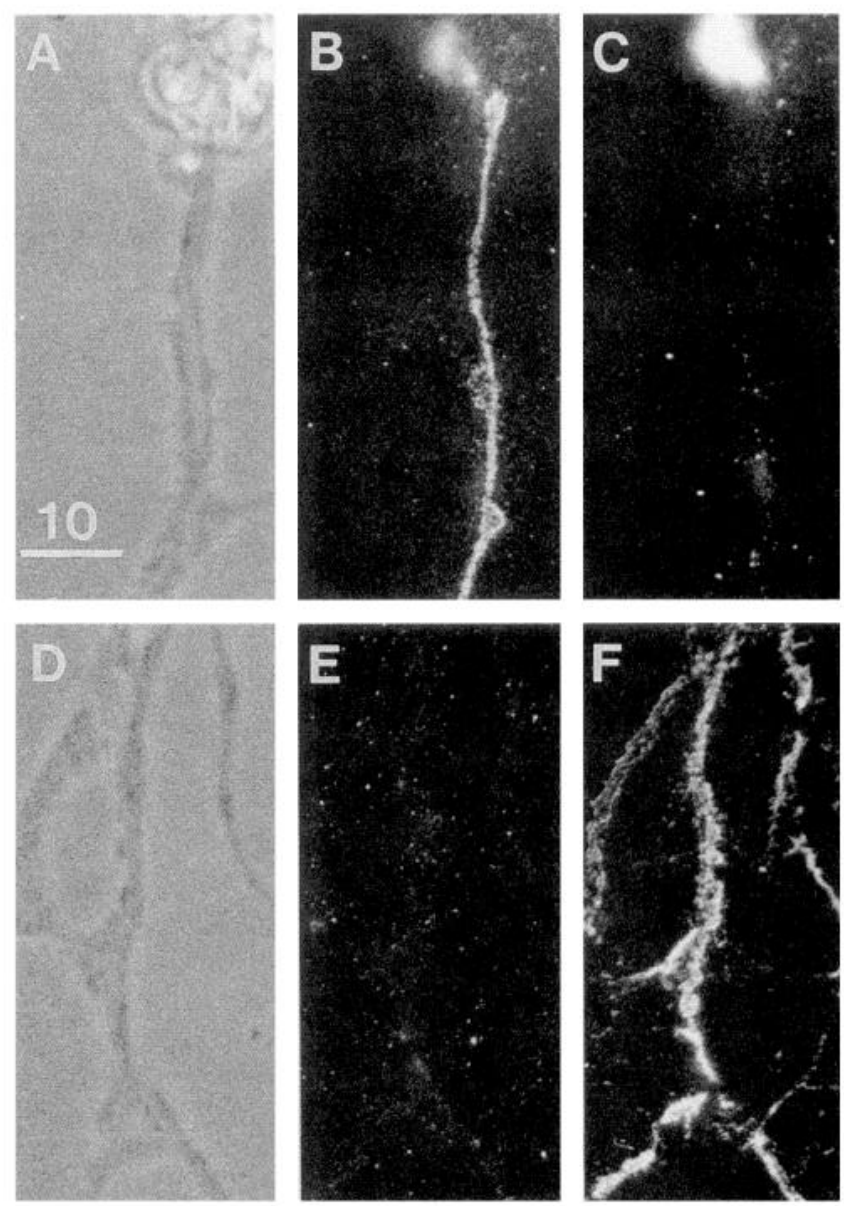

Figure 4. Specificity of agrin staining. $A-C$, From a culture stained with anti-36 and $\mathrm{B} 7$ followed by FGAR and RGAM. Along the neurite $(A)$ is anti-36 immunofluorescence in the focal plane of the substrate $(B)$ but no B7 immunofluorescence $(C)$. Some debris associated with the soma, and not in focus, was stained with both combinations of antibody. $D-F$, From a culture which was stained with pre-36 and $C 3$ followed by FGAR and RGAM. The neuritic pathway $(D)$ had no pre36 immunofluorescence $(E)$ but did exhibit $\mathrm{C} 3$ immunofluorescence in the focal plane of the substrate $(F)$. Scale bar, $10 \mu \mathrm{m}$.

after completely eliminating the neurites. An example is shown in Figure 6. After photographing the living neurites (Fig. 6 A) they were eliminated by a combination of aggressive rinsing followed by treatment with Triton X-100 (see Materials and Methods). The cell-free culture was then stained for agrin with C3, using RGAM as the second antibody. Figure $6 B$ shows the complete absence of the neurites and Figure $6 C$ shows the agrin immunofluorescence associated with much of the original neuritic pathway as well as fainter, more diffusely distributed immunofluorescence in the region where the cell bodies had been located. As expected, all of the immunofluorescence was in the same plane of focus as the culture substrate. In addition the patterns, intensity, and extensiveness of the agrin immunofluorescence in cultures whose neurites had been entirely elimi-

at the site from which the neurite retracted but not at the new position of the retracted neurite. Immunofluorescence is also apparent along the rest of the neuritic pathway, including the region of new growth (small arrowheads). The arrow points to a branch of immunofluorescence without a corresponding neurite. $D-I$, Higher-magnification views of the site of neuritic retraction $(D, E)$, of the branch of immunofluorescence without a neurite $(F, G)$, and of the region of new growth $(H, I)$. Note that the agrin immunofluorescence is at the same focal plane as the dots of fluorescence that were scattered on the surface of the substrate. Scale bars: $A-C, 20 \mu \mathrm{m} ; D-I, 10 \mu \mathrm{m}$. 

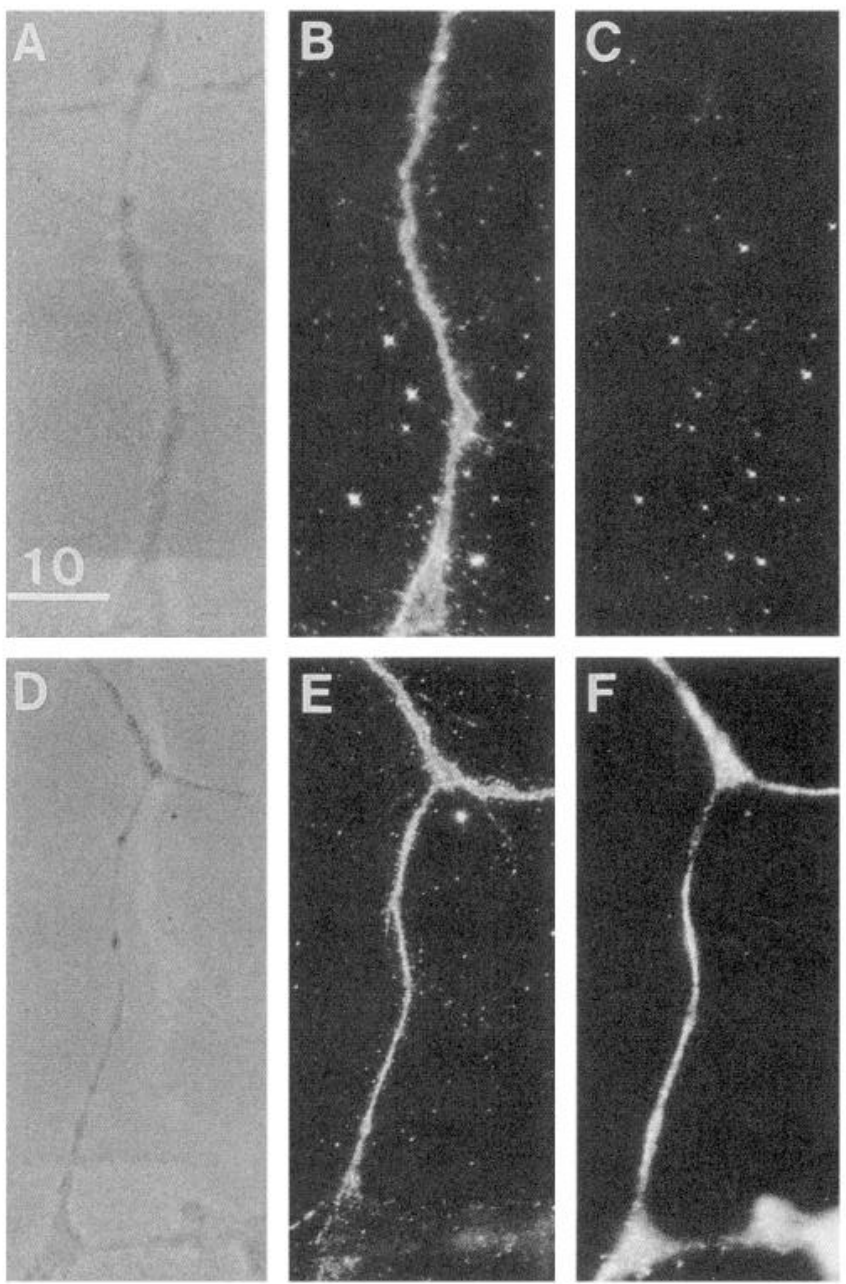

Figure 5. Staining for agrin compared to staining for tau $(\tau)$, a microtubule associated protein. $A-C$, A living culture was stained with $\mathrm{C} 3$ and anti- $\tau$ followed by FGAM and RGAR. Some of the neuritic pathway $(A)$ exhibited agrin immunofluorescence $(B)$ but $\tau$ immunofluorescence (C) was not detected. $D-F$, From a culture that was stained alive with C3 followed by FGAM, fixed and permeabilized with methanol, and then stained with anti- $\tau$ followed by RGAR. Most of the neuritic pathway $(D)$ exhibited agrin immunofluorescence $(E)$. The neurites exhibited $\tau$ immunofluorescence $(F)$ whether or not they externalized agrin. Scale bar, $10 \mu \mathrm{m}$.

nated were indistinguishable from those seen in cultures whose neurites were present. This was the case whether the cultures were stained before or after eliminating the neurites. In fact, in the experiment of Figure 6 the culture was stained not only with C3-RGAM after eliminating the neurites but also with anti-36FGAR before eliminating the neurites. The patterns of immunofluorescence obtained with both staining combinations were identical. Even when examined at high magnification, the relative intensity of the detailed substructure of the immunofluorescence obtained by staining after neurite elimination was identical to that obtained by staining prior to neurite elimination (Fig. 6D,E). The fact that staining after neurite elimination did not reveal any additional sites of agrin immunofluorescence indicates that only externalized agrin, and not any intracellular agrin, was left behind bound to the substrate.

That the neurites were entirely eliminated by our neurite elimination protocol was confirmed by two additional tests. First, immunofluorescent staining for intracellular proteins (micro-
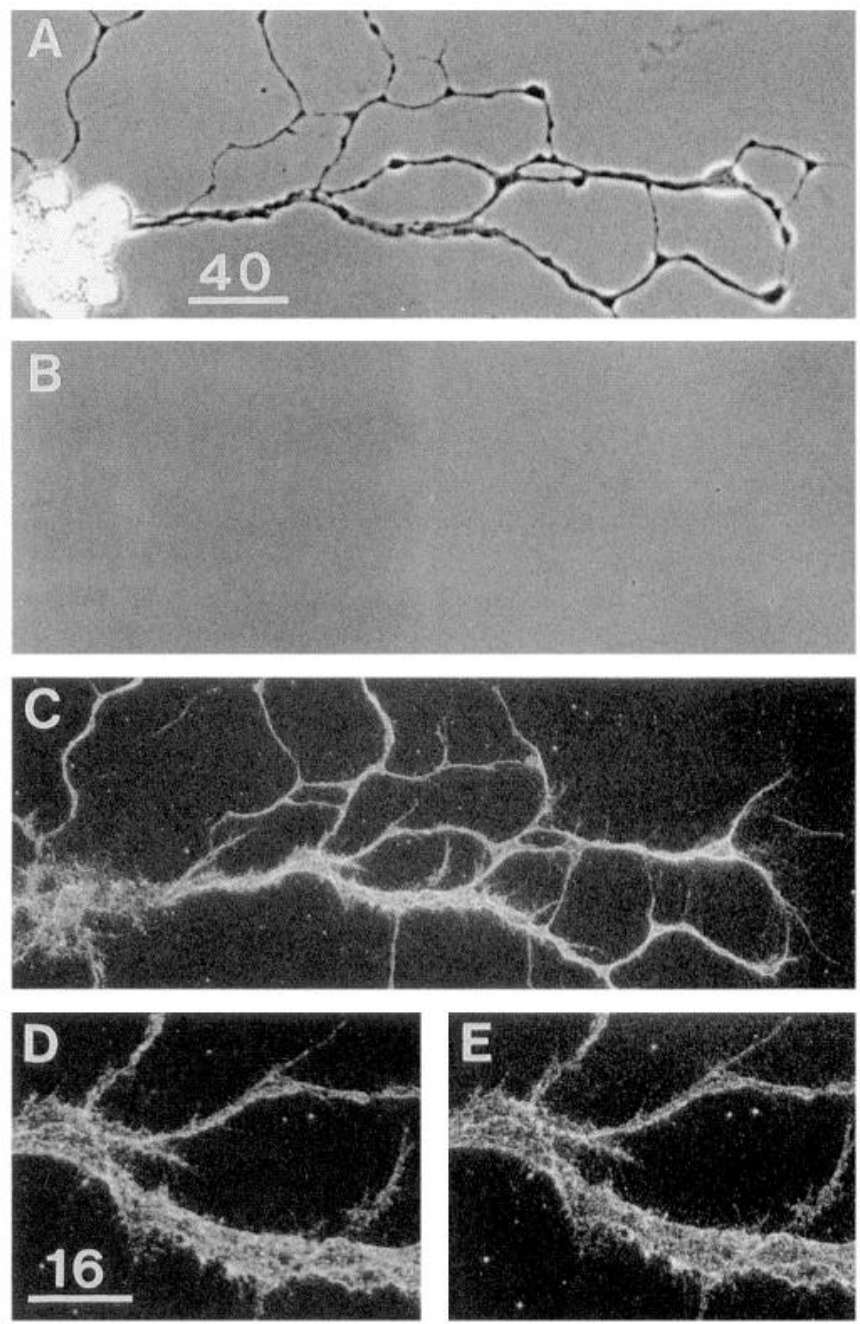

Figure 6. Agrin immunofluorescence following neurite elimination After photographing the living neurites $(A)$, they were eliminated $(B)$ and the culture was then stained with $\mathrm{C} 3$ followed by RGAM. Immunofluorescence $(C)$ was associated with most of the former neuritic pathway. A portion of the immunofluorescence is shown at higher magnification $(D)$. Before photographing $A$, the culture was stained with anti-36 followed by FGAR. The resulting immunofluorescence is shown in $E$ for the same portion of the field as $D$. The staining patterns are virtually identical. Subtle differences reflect a small shift in the focus between $D$ and $E$. Scale bars: $A-C, 40 \mu \mathrm{m} ; D$ and $E, 16 \mu \mathrm{m}$.

tubule-associated protein, synaptotagmin, and neurofilament protein) was entirely negative even when the concentration of primary antibody was fivefold greater than that required to obtain bright immunofluorescence when the neurites were present. Second, whereas bright NCAM immunofluorescence was observed over the entire surface of all neurites (agrin positive and agrin negative), none was detected when the staining for NCAM was carried out after neurite elimination (Fig. 7). This was the case even when the concentration of primary antibody was increased fivefold. Taken together, the results indicate that neuritic remnants were not left behind on the culture substrate following neurite elimination and that the agrin externalized by the SC neurites was bound tightly to the substrate.

\section{SC neurites on muscle cells}

Previously, when SC neurons were grown on a different culture substrate that did not bind significant amounts of externalized 
agrin, the agrin that was deposited on muscle cells was typically discontinuous and colocalized with sites of $\mathrm{AChR}$ accumulation (Cohen and Godfrey, 1992). Since, in the present study, agrin was deposited on the culture substrate in a continuous fashion along virtually the entire neuritic arbor of SC neurons, it was of interest to examine how the deposition of agrin would be affected when the same neurites grew across muscle cells. A typical example is shown in Figure 8. Where the neurites were in contact with the culture substrate the agrin immunofluorescence was continuous and where they grew over muscle cells it was highly discontinuous and colocalized with sites of AChR accumulation. When the neurites returned to the culture substrate the immunofluorescence became continuous again. By contrast, NCAM immunofluorescence was present along the entire length of most neurite-muscle contacts, thereby indicating that the antibodies had access to synaptic as well as nonsynaptic portions of contact (Fig. 9). It is apparent from these results that the muscle cells have a profound local influence on the pattern of agrin deposition by SC neurites. It is also apparent from the example of Figure 8 that the intensity of the immunofluorescence tended to be greater on the culture substrate than at some of the sites of agrin deposition along neurite-muscle contacts.

\section{Neuronal specificity in the externalization of agrin}

Agrin mRNA has a widespread distribution in the rat and chick nervous systems and is present even in dorsal root ganglia (Rupp et al., 1991; Horton et al., 1992; McMahan et al., 1992). Yet when Xenopus DRG neurites contact muscle cells they fail to induce a local accumulation of AChRs (Cohen and Weldon, 1980; Kidokoro et al., 1980). This suggests that Xenopus DRG neurites may not externalize significant quantities of agrin. To test this point we stained DRG cultures for agrin. As shown in Figure 10 there was essentially no detectable immunofluorescence along the DRG neurites, either on the culture substrate or along paths of contact with muscle cells. Nor was there any detectable AChR accumulation along the DRG neurite-muscle contacts. Sometimes, faint immunofluorescence was seen in regions of cell bodies but it quickly declined below background levels beyond these regions. A similar paucity of immunofluorescence was observed when DRG cultures were stained after neurite elimination. Overall, these results indicate that Xenopus DRG neurons externalized little if any agrin along their neuritic outgrowth.

\section{Discussion}

This study has provided a direct demonstration of agrin deposition along the entire neuritic outgrowth of competent SC neurons. It has also revealed that muscle cells profoundly influence the pattern of agrin deposition by SC neurites. Furthermore, the lack of agrin deposition along the neuritic outgrowth of DRG neurons, and the failure of these neurons to induce AChR accumulation along their neuritic contacts with muscle cells, adds to the evidence that agrin is the primary neural agent mediating nerve-induced aggregation of AChRs during nervemuscle synaptogenesis.

These new insights depended largely on the use of a culture substrate that bound tightly the agrin externalized by SC neurons. As a consequence of this tight binding, externalized agrin was observed not only in the presence of neurites but also along former neuritic pathways from which the neurites had retracted spontaneously or in response to experimental manipulation. The substrate consisted of a combination of rat tail collagen
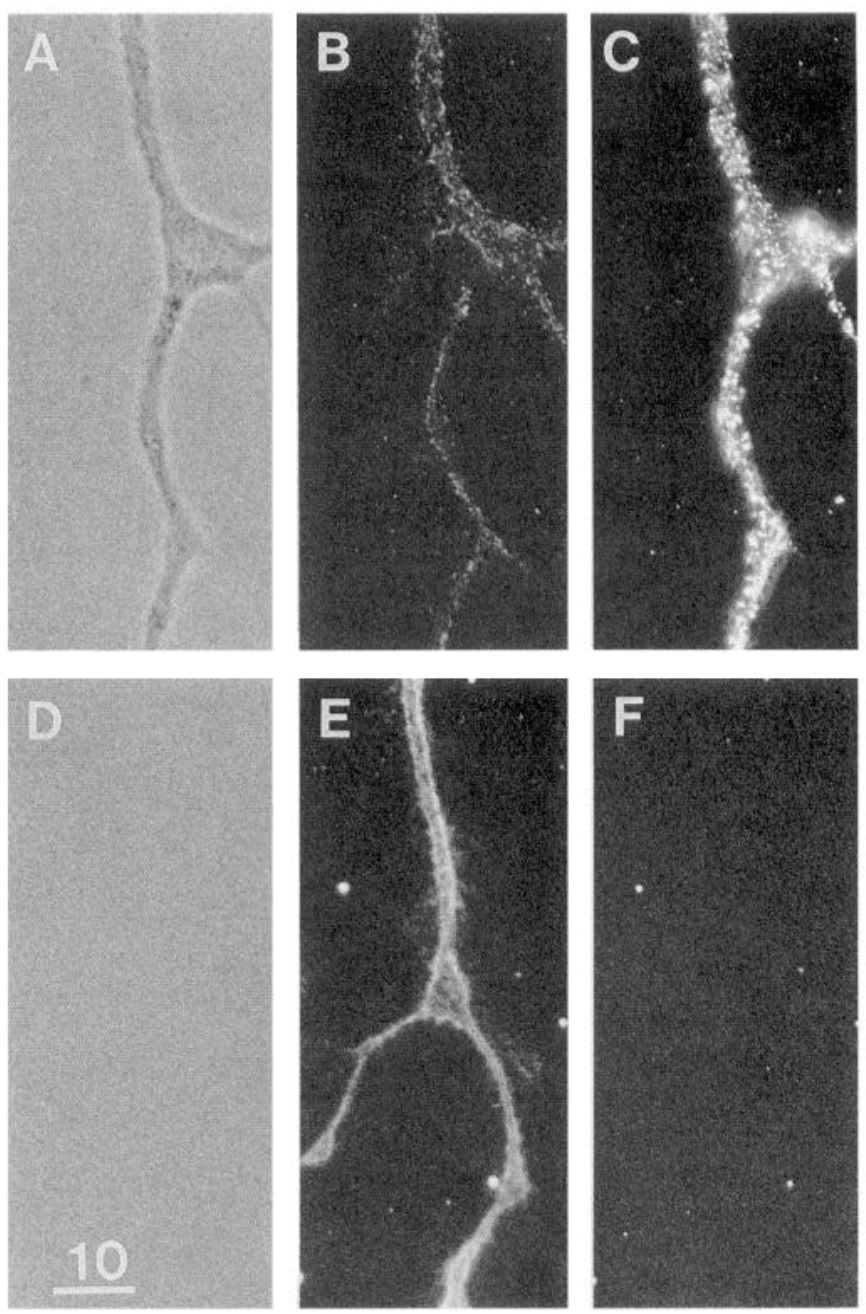

Figure 7. Staining for agrin compared to staining for NCAM. $A-C$, From a culture that was fixed for $10 \mathrm{~min}$ with $4 \%$ formaldehyde and then stained with C3 and anti-NCAM followed by FGAM and RGAR. The neuritic pathway $(A)$ has faint agrin immunofluorescence at the same focal plane as the substrate $(B)$. NCAM immunofluorescence is also present at the neurite-substrate interface as well as over the rest of the surface of the neurite $(C) . D-F$, From a culture that, after neurite elimination, was stained with $\mathrm{C} 3$ and anti-NCAM followed by FGAM and RGAR. Former neuritic pathways $(D)$ exhibited agrin immunofluorescence $(E)$ but no NCAM immunofluorescence $(F)$. Scale bar, 10 $\mu \mathrm{m}$.

(type I) and mouse tumor entactin, collagen (type IV), and laminin. Some of these components are found in basal laminae and agrin is bound tightly to the synaptic basal lamina of mature neuromuscular junctions (Reist et al., 1987). Evidence that the agrin externalized by appropriately transfected COS cells binds to a culture substrate of basal lamina derived from embryonic chick retina further emphasizes the importance of basal lamina components in binding agrin (McMahan, 1990). In addition, in embryonic muscle, the agrin immunofluorescence that is associated with AChR aggregates is always colocalized with immunofluorescence for laminin and heparan sulfate proteoglycan (Godfrey et al., 1988). But which of the components confer to the substrate its agrin-binding property and whether they also participate in the binding of agrin at nerve-muscle synapses are questions that remain to be investigated. That collagen IV or entactin may play a role is suggested by the lack of substratebound agrin when Xenopus nerve and muscle cells were cultured 
Figure 8. Agrin immunofluorescence along neurite-substrate and neuritemuscle contacts. A, A composite photograph showing a neurite that left the substrate to grow on a muscle cell and then returned to the substrate. Arrowheads mark the points of transition of neuritic growth on the substrate and on the muscle cell. $B$, The corresponding agrin immunofluorescence. $C$, The corresponding $A C h R$ accumulation. Note the pattern of interrupted immunofluorescence, and colocalized AChR accumulation, along the neurite-muscle contact and the continuous immunofluorescence along the neurite-substrate contact. Portions of the neuritic growth and fluorescent staining on the muscle cell are out of focus. Some nonspecific fluorescence was associated with muscle cell granules (upper right). Scale bar, $10 \mu \mathrm{m}$.
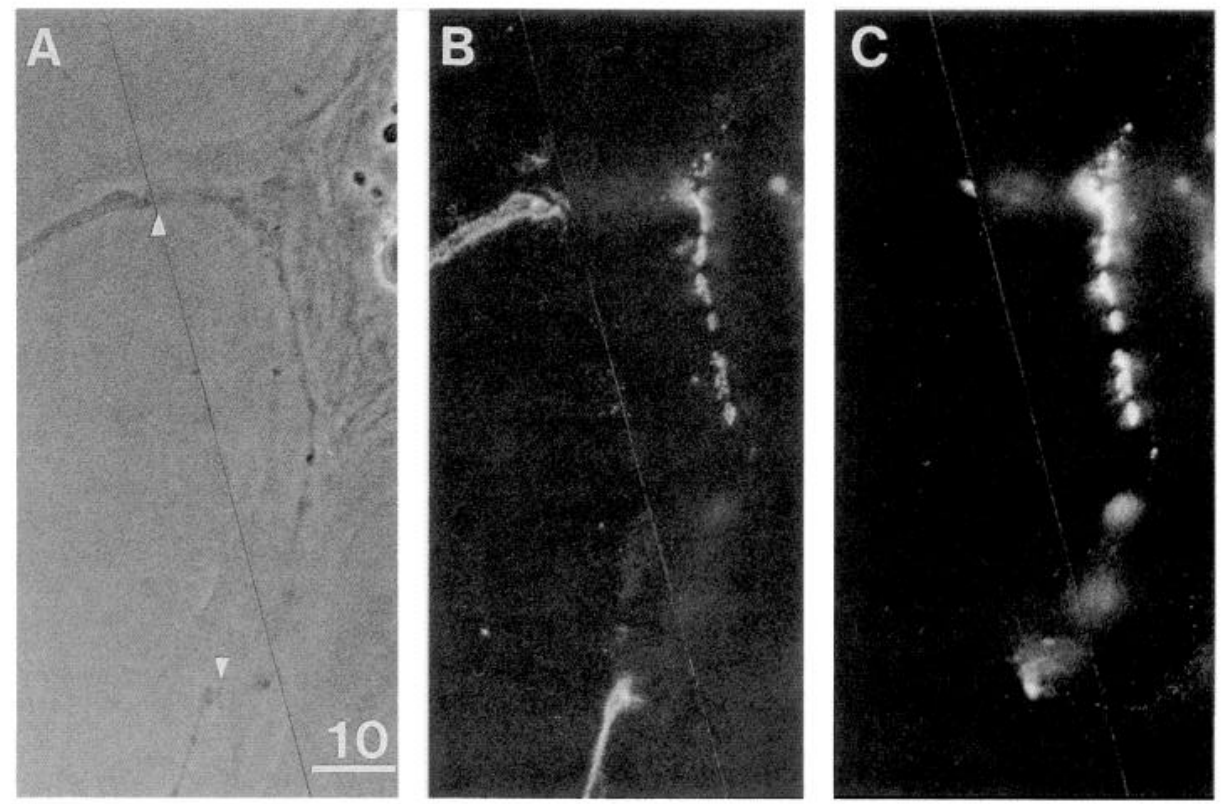

Unlike externalized agrin, externalized NCAM did not remain associated with the culture substrate following neurite elimination. This is not unexpected since NCAM and agrin share little molecular homology (Lander, 1989; McMahan et al., 1992). Moreover, neurons often express transmembrane isoforms of NCAM (Rutishauser and Jessell, 1988) whereas neural agrin does not appear to be an integral membrane protein (Rupp et al., 1991; Tsim et al., 1992). What is apparent is that surface membrane was not left on the substrate when the neurites were experimentally eliminated. Any linkage between externalized agrin and the surface membrane of neurites must be relatively weak compared to agrin's binding affinity for the culture substrate.

Presumably the SC neurons that deposited agrin along their neuritic outgrowth were motor neurons. That they were in the majority is consistent with previous findings that a majority of Xenopus SC neurons in culture can induce AChR aggregation and establish functional synapses on muscle cells (Anderson et al., 1977; Cohen et al., 1987). Furthermore, studies on chick have indicated that motor neurons are enriched in agrin and its mRNA (Magill-Solc and McMahan, 1988; Tsim et al., 1992) and that they are the only SC neurons that induce AChR aggregation (Role et al., 1985). Also of relevance to the question of neuronal specificity in the externalization of agrin is our finding that, unlike SC (motor) neurons, DRG neurons deposited little if any agrin along their neuritic outgrowth. This correlates with the neuronal specificity previously established for the neurite-induced aggregation of AChRs on muscle cells (Cohen and Weldon, 1980; Kidokoro et al., 1980). Taken together, these results add to the evidence (Cohen and Godfrey, 1992; McMahan et al., 1992; Reist et al., 1992) that neural agrin is a primary synaptogenic agent.

On the other hand, it is not clear at present how to reconcile the lack of agrin deposition along the neuritic outgrowth of Xenopus DRG neurites with the presence of significant levels of agrin mRNA in rat and chick DRG (Horton et al., 1992; Rupp et al., 1992). Perhaps DRG neurons have low levels of agrin protein despite their agrin mRNA content or Xenopus DRG neurons in culture differ in these respects from rat and
Figure 9. NCAM immunofluorescence along synaptic and nonsynaptic portions of a neurite-muscle contact. $A$, A neurite in contact with the edge of a muscle cell. The short portion of neurite above the arrow is out of focus and not in contact with the muscle cell. $B$, The corresponding NCAM immunofluorescence. $C$, The corresponding AChR fluorescence. The three photographs were taken at the same focus, optimal for the AChR aggregates, using an oil immersion $100 \times$ objective. The NCAM immunofluorescence was associated not only with the free surface of the neurite but also with the surface in contact with the muscle cell, both at and away from sites of AChR accumulation. Scale bar, 5 $\mu \mathrm{m}$. 

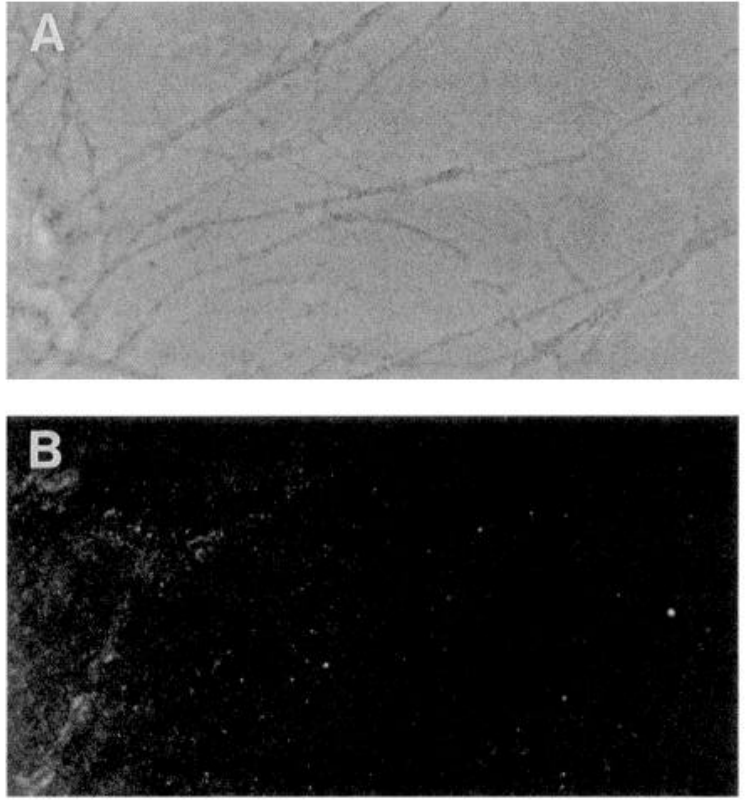
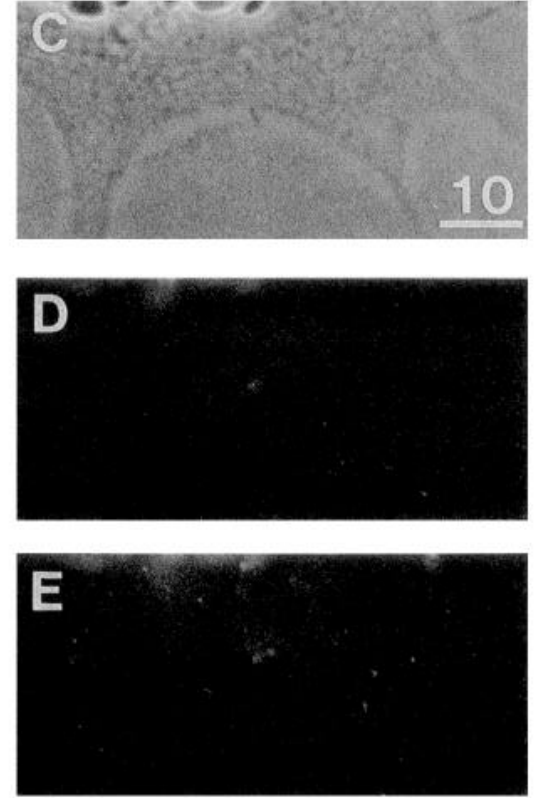

Figure 10. Agrin immunofluorescence in DRG cultures. $A$ and $B$, From a culture stained with anti-36 and $\mathrm{C} 3$ followed by FGAR and FGAM. Faint immunofluorescence is apparent in the region of the cell bodies but not along the rest of their neuritic outgrowth. $C-$ $E$, From a culture of DRG neurons and muscle cells that was stained for AChRs and for agrin with anti-36 and C3 followed by FGAR and FGAM. The neurite-muscle contacts $(C)$ exhibited no immunofluorescence $(D)$ or AChR accumulation $(E)$. Scale bar, $10 \mu \mathrm{m}$. chick DRG neurons. Alternatively, it may be that DRG neurons process agrin differently from SC neurons and this difference affects the delivery of agrin to the neurites, its externalization, or its affinity for binding to muscle cells and culture substrate. This would be an additional means, besides alternative splicing (Ferns et al., 1991; Ruegg et al., 1992; Smith et al., 1992), for modifying the functional role of agrin in different types of neurons.

That the deposition of agrin typically extended to the limits of the SC neuritic outgrowth can be explained by assuming that agrin is externalized in the region of the growth cones. Such an assumption is in line with previous evidence that a variety of proteins are externalized in the region of nerve growth cones (Pittman, 1985; Wood et al., 1992). But interestingly, the observation of reduced agrin immunofluorescence in regions of new neuritic growth raises the possibility that externalized agrin may continue to accumulate at a particular site on the substrate for some time after the growth cone has passed that site. One way this could occur would be if externalized agrin molecules are initially linked to the surface of the neurite and move in the plane of the membrane. These mobile, surface-linked molecules would thus move away from the site where they were externalized and would become immobilized only upon contacting the substrate some time later. Such a "diffusion-trap mechanism" is analogous to that which is associated with the aggregation of AChRs on the surface of muscle cells (Anderson and Cohen, 1977; Ziskind-Conhaim et al., 1984; Kidokoro et al., 1986). It would also account for the sharp borders of the substrate-bound agrin and the increased accumulation of agrin that was sometimes seen at the edges of broad portions of neurites. In moving around the circumference of the neurite, mobile agrin molecules on the free surface of the neurite would tend to first encounter the substrate at the outermost edge of the neuritesubstrate contact. Accordingly, this would be the site of highest probability for agrin molecules to be trapped by binding to the substrate. During nerve-muscle synaptogenesis appropriate molecules on the surface of the muscle cell would act as the trap for the mobile agrin molecules on the surface of the growing axon. Alternative explanations for the sharp borders of the sub- strate-bound agrin are that the agrin was preferentially externalized at the sites of neurite-substrate contact or that at these sites the rate of dissociation of agrin from the substrate was reduced because of an additional linkage to the neurite.

Whereas the deposition of agrin was essentially continuous along the length of neurites on the culture substrate, it became discontinuous where the neurites contacted muscle cells. In view of the evidence that neural agrin is a primary synaptogenic agent this marked influence of muscle cells on the pattern of agrin deposition indicates that some of their properties limit the extensiveness of agrin deposition and synapse formation on their surface. This conclusion is in line with previous results which indicate that the capacity of individual muscle cells to develop synaptic specializations can be saturated (Cohen et al., 1987). What these limiting factors are can only be the subject of speculation at present. One obvious possibility is that the number of agrin binding sites on embryonic muscle cells is limiting. Such a possibility is consistent not only with the interrupted pattern of agrin deposition on muscle cells but also with the observation that the agrin immunofluorescence along neuritemuscle contacts was not always as bright as that on the culture substrate.

Discontinuities in agrin deposition along neurite-muscle contacts have been observed in cocultures ranging from $7.5 \mathrm{hr}$ to $4 \mathrm{~d}$ in age and even along contacts as young as $90 \mathrm{~min}$ (Cohen and Godfrey, 1992). To account for these discontinuities it is pertinent to know (1) the distribution of agrin binding sites on the muscle cell surface immediately prior to contact with the neurite and (2) whether changes occur in this distribution following neurite-muscle contact. Such information is not available for the case of Xenopus nerve-muscle cultures but it is known that basal lamina molecules such as heparan sulfate proteoglycan, which may bind neural agrin (Wallace, 1990), have a nonuniform distribution on Xenopus muscle cells as well as on chick muscle cells in culture (Anderson and Fambrough, 1983; Bayne et al., 1984). On the other hand, tests with partially purified Torpedo agrin have suggested that its binding sites on chick muscle cells in culture are uniformly distributed initially and then cluster at sites of agrin-induced AChR aggregation 
(Nastuk et al., 1991). Such a redistribution of agrin binding sites might also occur along paths of neurite-muscle contact during synapse formation. Presumably, once synapse formation is initiated at specific sites, the neuritic externalization of agrin becomes preferentially redirected to those sites as a result of a retrograde interaction with the muscle cells. In addition, it is known that muscle-derived proteins including a heparan sulfate proteoglycan become incorporated in the developing basal lamina at newly forming synaptic sites (Swenarchuk et al., 1990). If such molecules bind neural agrin they could enhance its retention at these sites compared to neighboring nonsynaptic sites along the path of neurite-muscle contact.

The local externalization, binding, and retention of neural agrin may play an important role as well in the competitive interactions between neurons during synapse development. For example, the elimination of polyneuronal innervation at developing neuromuscular junctions involves a disappearance of some sites of aggregated AChRs from the synapses (Balice-Gordon and Lichtman, 1993). Is this disappearance preceded by a loss of neural agrin at those sites? It may also be pertinent that there was variability in the intensity of the agrin immunofluorescence associated with different neuritic arbors and with different portions of individual arbors. Differences in the amounts of agrin externalized by different $\mathrm{SC}$ neurons may account for the previous finding that individual SC neurons in culture exhibit about a fourfold range in their capacity to induce AChR aggregation along their neurite-muscle contacts (Cohen et al., 1987). If individual motor neurons in vivo also differ in the amounts of agrin their axon terminals externalize, such differences would likely be important determinants in their competitive interactions.

\section{References}

Anderson MJ, Cohen MW (1977) Nerve-induced and spontaneous redistribution of acetylcholine receptors on cultured muscle cells. J Physiol (Lond) 268:757-773.

Anderson MJ, Fambrough DM (1983) Aggregates of acetylcholine receptors are associated with plaques of a basal lamina heparan sulfate proteoglycan on the surface of skeletal muscle fibers. J Cell Biol 97: 1396-1411.

Anderson MJ, Cohen MW, Zorychta E (1977) Effects of innervation on the distribution of acetylcholine receptors on cultured amphibian muscle cells. J Physiol (Lond) 268:731-756.

Balice-Gordon RJ, Lichtman JW (1993) In vivo observations of preand postsynaptic changes during the transition from multiple to single innervation at developing neuromuscular junctions. J Neurosci 13: 834-855.

Bayne EK, Anderson MJ, Fambrough DM (1984) Extracellular matrix organization in developing muscle: correlation with acetylcholine receptor aggregates. J Cell Biol 99:1486-1501.

Chow I, Cohen MW (1983) Developmental changes in the distribution of acetylcholine receptors in the myotomes of Xenopus laevis. J Physiol (Lond) 339:553-571.

Cohen MW, Godfrey EW (1992) Early appearance of and neuronal contribution to agrin-like molecules at embryonic frog nerve-muscle synapses formed in culture. J Neurosci 12:2982-2992.

Cohen MW, Weldon PR (1980) Localization of acetylcholine receptors and synaptic ultrastructure at nerve-muscle contacts in culture: dependence on nerve type. J Cell Biol 86:388-401.

Cohen MW, Rodriguez-Marin E, Wilson EM (1987) Distribution of synaptic specializations along isolated motor units in Xenopus nervemuscle cultures. J Neurosci 7:2849-2861.

Cohen MW, Moody-Corbett F, Godfrey EW (1992) Externalization of agrin-like molecules by embryonic neurons. Soc Neurosci Abstr 18:208.

Ferns M, Hoch W, Campanelli JT, Rupp F, Hall 7.W, Scheller RH (1992) RNA splicing regulates agrin-mediated acetylcholine receptor clustering activity on cultured myotubes. Neuron 8:1079-1086.
Frank E, Fischbach GD (1979) Early events in neuromuscular junction formation in vitro. Induction of acetylcholine receptor clusters in the postsynaptic membrane and morphology of newly formed synapses. J Cell Riol 83:143-158.

Godfrey EW (1991) Comparison of agrin-like proteins from the extracellular matrix of chicken kidney and muscle with neural agrin, a synapse organizing protein. Exp Cell Res 195:99-109.

Godfrey EW, Siebenlist RE, Wallskog PA, Walters LM. Bolender DL, Yorde DE (1988) Basal lamina components are concentrated in premuscle masses and at early acetylcholine receptor clusters in chick embryo hindlimb muscles. Dev Biol 130:471-486.

Hayes BP, Roberts A (1973) Synaptic junction development in the spinal cord of an amphibian embryo: an electron microscope study. Z Zellforsch Mikrosk Anat 137:251-269.

Horton SE, Ruegg MA, Escher G, Kröger S. McMahan UJ (1992) Differential distribution of members of the agrin protein family in the nervous system. Soc Neurosci Abstr 18:208.

Jacobson M, Rutishauser U (1986) Induction of neural cell adhesion molecule (NCAM) in Xenopus embryos. Dev Biol 116:524-531.

Kidokoro Y, Anderson MJ, Gruener R (1980) Changes in synaptic potential properties during acetylcholine receptor accumulation and neurospecific interactions in Xenopus nerve-muscle cell culture. Dev Biol 78:464-483.

Kidokoro $\mathrm{Y}$, Brass B, Kuromi $\mathrm{H}$ (1986) Concanavalin A prevents acetylcholine receptor redistribution in Xenopus nerve-muscle cultures. J Neurosci 6:1941-1951.

Kullberg RW, Lentz TL, Cohen MW (1977) Development of the myotomal neuromuscular junction in Xenopus laevis: an electrophysiological and fine-structural study. Dev Biol 60:101-129.

Lander AD (1989) Understanding the molecules of neural cell contacts: emerging patterns of structure and function. Trends Neurosci 12:189-195.

Magill-Solc C, McMahan UJ (1988) Motor neurons contain agrin-like molecules. J Cell Biol 107:1825-1833.

Matthew WD, Tsavcler L, Rcichardt LF (1981) Identification of a synaptic vesicle-specific membrane protein with a wide distribution in neuronal and neurosecretory tissue. J Cell Biol 91:257-269.

McMahan UJ (1990) The agrin hypothesis. Cold Spring Harbor Symp Quant Biol 55:407-418.

McMahan UJ, Horton SE, Werle MJ, Honig LS, Kröger S, Ruegg MA, Escher $G$ (1992) Agrin isoforms and their role in synaptogenesis. Curr Opin Cell Biol 4:869-874.

Moody-Corbett F, Cohen MW (1981) Localization of cholinesterase at sites of high acetylcholine receptor density on embryonic amphibian muscle cells cultured without nerve. J Neurosci 1:596-605.

Moody-Corbett F, Cohen MW (1982) Influence of nerve on the formation and survival of acetylcholine receptor and cholinesterase patches on embryonic Xenopus muscle cells in culture. J Neurosci 2:633-646.

Nastuk MA, Lieth E, Ma J, Cardasis CA, Moynihan EB, McKechnie BA, Fallon JR (1991) The putative agrin receptor binds ligand in a calcium-dependent manner and aggregates during agrin-induced acetylcholine receptor clustering. Neuron 7:807-818.

Nieuwkoop PD, Faber J (1967) Normal table of Xenopus laevis (Daudin), 2d ed. Amsterdam: North Holland.

Pittman RN (1985) Release of a plasminogen activator and a calciumdependent metalloprotease from cultured sympathetic and sensory neurons. Dev Biol 110:91-101.

Pollerberg GE, Schachner M, Davoust J (1986) Differentiation statedependent surface mobilities of two forms of the neural cell adhesion molecule. Nature 324:462-465.

Reist NE, Magill C, McMahan UJ (1987) Agrin-like molecules at synaptic sites in normal, denervated, and damaged skcletal muscles. J Cell Biol 105:2457-2469.

Reist NE, Werle MJ, McMahan UJ (1992) Agrin released by motor neurons induces the aggregation of acetylcholine receptors at neuromuscular junctions. Neuron 8:865-868.

Role LW, Matossian VR, O'Brien RJ, Fischbach GD (1985) On the mechanism of acetylcholine receptor accumulation at newly formed synapses on chick myotubes. J Neurosci 5:2197-2204.

Ruegg MA, Tsim KWK, Horton SE, Kröger S, Escher G, Gensch EM, McMahan UJ (1992) The agrin gene codes for a family of basal lamina proteins that differ in function and distribution. Neuron 8:691699.

Rupp F, Payan DG, Magill-Solc C, Cowan DM, Scheller RH (1991) Structure and expression of a rat agrin. Neuron 6:811-823. 
Rutishauser U, Jessell TM (1988) Cell adhesion molecules in vertebrate neural development. Physiol Rev 68:819-857.

Smith MA, Magill-Solc C, Rupp F, Yao MY-M, Schilling JW, Snow P, McMahan UJ (1992) Isolation and characterization of a cDNA clone that encodes an agrin homolog in the marine ray. Mol Cell Neurosci 3:406-417.

Südhof TC, Jahn R (1991) Proteins of synaptic vesicles involved in exocytosis and membrane recycling. Neuron 6:665-677.

Swenarchuk LE, Champaneria S, Anderson MJ (1990) Induction of a specialized muscle basal lamina at chimaeric synapses in culture. Development 110:51-61.
Tsim KWK, Ruegg MA, Escher G, Kröger S, McMahan UJ (1992) cDNA that encodes active agrin. Neuron 8:677-689.

Wallace BG (1990) Inhibition of agrin-induced acetylcholine receptor aggregation by heparin heparan sulfate, and other polyanions. J Neurosci 10:3576-3582.

Wood MR, DeBin J, Strichartz GR, Pfenninger KH (1992) Plasmalemmal insertion and modification of sodium channels at the nerve growth cone. J Neurosci 12:2948-2959.

Ziskind-Conhaim L, Geffen I, Hall ZW (1984) Redistribution of acetylcholine receptors on developing rat myotubes. J Neurosci 4:23462349. 